\title{
Rellich-type Discrete Compactness for Some Discontinuous Galerkin FEM *
}

\author{
Fumio KIKUCHI \\ Graduate School of Economics, Hitotsubashi University \\ 2-1 Naka, Kunitachi, Tokyo, 186-8601 Japan \\ E-mail: fkikuchi@econ.hit-u.ac.jp, kikuchi@ms.u-tokyo.ac.jp
}

April 14, 2011

\begin{abstract}
We deduce discrete compactness of Rellich type for some discontinuous Galerkin finite element methods (DGFEM) including hybrid ones, under fairly general settings on the triangulations and the finite element spaces. We make use of regularity of the solutions to an auxiliary second-order elliptic boundary value problem as well as the error estimates of the associated finite element solutions. The present results can be used for analyzing DGFEM applied to some boundary value and eigenvalue problems, and also to derive the discrete Poincaré-Friedrichs inequalities.
\end{abstract}

Keywords : discontinuous Galerkin FEM, polygonal element, discrete compactness, Rellich's selection theorem

Mathematical Subject Classification (2000) : 65N30, 65N12

\section{Introduction}

In recent years, much attention has been drawn to the discontinuous Galerkin finite element methods (DGFEM) [4, $9,16,24]$. They use discontinuous approximate functions, where the discontinuity is dealt with by the interelement Lagrange multiplier and/or interior penalty methods. Such methods have a merit that various approximate functions besides the usual piecewise interpolation polynomials can be used, since the inter-element continuity and unisolvence conditions are much relaxed. In particular, they are expected to be more flexible in element shapes than classical FEM.

It is to be noted here that they are closely related to the non-conforming and hybrid methods, which use discontinuous approximate functions, and the latter of which is characterized by the use of inter-element Lagrange multipliers [28]. Simplifying the hybrid displacement method of Tong [14, 31], the present author and his coauthor developed some finite elements in a series of papers e.g. [20,21], but such an attempt got only partial success because of lack of effective stabilization methods [25]. In this respect, the interior penalty approach [2] is now recognized to be crucial in both handling with the inter-element discontinuity and assuring numerical stability.

Stimulated by rapid development of DGFEM, the present author and his coworkers proposed a hybrid displacement type DGFEM by stabilizing the above old method using the interior penalty technique. They showed the idea for the 2D Poisson equation and the plane stress problem with reasonable numerical results $[22,27]$. It turned out that such an approach is actually available as a finite element method by appropriate choice of the stabilization coefficients, and it is fairly robust to deformation of element shape. Moreover, it can be used just like the conventional finite element methods: usual element-by-element procedures are

* This work was in part supported by JSPS, Grant-in-Aid for Scientific Research (C) 19540115. 
available, and mixed use with the conventional elements is possible. See also $[12,13]$ for a closely related approaches.

Since such formulations make full use of discontinuous approximate functions strongly dependent on triangulations, some standard tools in numerical functional analysis may not be available in their genuine forms. An important example of such techniques and results is the Rellich selection theorem [10, 30], which states that any weakly convergent sequence in $H^{1}(\Omega)$ (or its closed subspace) for the bounded Lipschitz domain $\Omega$ is strongly convergent in $L^{2}(\Omega)$. This theorem and similar ones are frequently employed for spectral and resolvent analyses of elliptic operators such as the Laplacian, and also for analysis of linear or semi-linear Poisson-like problems with lower-order linear or nonlinear terms. Moreover, the results obtained by such analyses are directly applicable to the related $H^{1}$-conforming finite elements.

On the other hand, if we want to use similar arguments in DGFEM, which are not $H^{1}$-conforming in principle, we must establish appropriate discrete analogs of the Rellich theorem [10, 30]. Such discrete compactness properties have played important roles especially in the analysis of edge finite elements for electromagnetics [7, 18, 19]. Moreover, difficulties of DGFEM applied to Laplace and various spectral problems are discussed e. g. in $[3,16]$, and are essentially related to the discrete compactness.

In this paper, we will first explain some DGFEM for 2D Poisson-like problems including hybrid ones. In particular, the interior penalty term and the lifting operator are introduced to the bilinear forms to assure numerical stability of the associated DGFEM $[4,12,13,26]$. For simplicity, we only consider symmetric bilinear forms, and omit the analysis of non-symmetric DGFEM. Then we discuss the discrete compactness properties of Rellich type, which will play important roles in numerical analysis of DGFEM. We will prove our main results under some popular hypotheses (cf. e.g. [4, 9]), with some observations on sufficient conditions for such hypotheses.

Some related results are also reported in e.g. [3,6], in which various discrete compactness properties are derived from some discrete Poincaré-Friedrichs inequalities. On the contrary, our method utilizes some error estimates of the solutions of auxiliary problems with regularity, and is completely within the framework of the Hilbert space method. It is also analogous to the methods previously used by the present author to derive discrete compactness for the edge finite elements [18, 19], and may be effective in some cases, although it might be difficult to apply to non-Hilbertian cases.

\section{Preliminaries and DGFEM}

\subsection{Auxiliary problem and notations}

Let $\Omega \subset \boldsymbol{R}^{2}$ be a bounded polygonal domain with boundary $\partial \Omega$. For $\Omega$, we can define the Hilbertian Sobolev spaces $L^{2}(\Omega)$ and $H^{\kappa}(\Omega)(\kappa>0)$, where the fractional cases $(\kappa \notin N)$ are included [5, 9]. The inner products of both $L^{2}(\Omega)$ and $L^{2}(\Omega)^{2}$ are designated by $(\cdot, \cdot)_{\Omega}$, with the associated norms done by $\|\cdot\|_{\Omega}$. Furthermore, the norms and the standard semi-norm of $H^{\kappa}(\Omega)$ are denoted by $\|\cdot\|_{\kappa, \Omega}$ and $|\cdot|_{\kappa, \Omega}$, respectively, where $|v|_{\kappa, \Omega}^{2}=\|v\|_{\kappa, \Omega}^{2}-\|v\|_{\kappa^{*}, \Omega}^{2}$ for $v \in H^{\kappa}(\Omega)\left(\kappa^{*}=[\kappa]\right.$ for $\kappa \notin N$ and $\kappa^{*}=\kappa-1$ for $\left.\kappa \in \boldsymbol{N}\right)$. For these spaces associated to domains other than $\Omega$, the same notations of spaces, norms etc. will be used with $\Omega$ replaced appropriately.

Let us consider a subset $\partial \Omega_{D}$ of $\partial \Omega$, which either is empty or consists of finitely many closed segments. Then we introduce a closed subspace $H_{D}^{1}(\Omega)$ of $H^{1}(\Omega)$ by

$$
H_{D}^{1}(\Omega)=\left\{v \in H^{1}(\Omega) ; v=0 \text { on } \partial \Omega_{D}\right\}
$$

which reduces to $H^{1}(\Omega)$ and $H_{0}^{1}(\Omega)$ respectively when $\partial \Omega_{D}=\emptyset$ and $\partial \Omega_{D}=\partial \Omega$.

Using this space, let us define an auxiliary boundary value problem : given $f \in L^{2}(\Omega)$, find $u \in H_{D}^{1}(\Omega)$ such that

$$
(\nabla u, \nabla v)_{\Omega}+(u, v)_{\Omega}=(f, v)_{\Omega} ; \forall v \in H_{D}^{1}(\Omega),
$$

where $\nabla$ denotes the gradient operator. The corresponding partial differential equation is $-\Delta u+u=f$, and the boundary conditions on $\partial \Omega$ and $\partial \Omega \backslash \partial \Omega_{D}$ are the homogeneous Dirichlet and Neumann ones, respectively. If we omit the term $(u, v)_{\Omega}$ in (2), we have the popular Poisson problem. 
The uniqueness and existence of $u$ of (2) in $H_{D}^{1}(\Omega)$ are trivial, and $u$ also belongs to $H^{\frac{3}{2}}+\sigma(\Omega)$ for a certain $\sigma \in] 0, \frac{1}{2}$ ] along with the estimation

$$
\|u\|_{\frac{3}{2}+\sigma, \Omega} \leq C_{D}\|f\|_{\Omega}
$$

where $C_{D}>0$ is a constant independent of $f$, cf. [15]. Thus the traces of $u$ and $\nabla u$ to any piecewise smooth $\operatorname{arc} \gamma \subset \bar{\Omega}$ (= closure of $\Omega$ ) are well-defined as functions in $L^{2}(\gamma)$ and $L^{2}(\gamma)^{2}$, respectively.

\subsection{Definitions and notations for triangulations}

We first construct a family of triangulations $\left\{\mathscr{T}^{h}\right\}_{h>0}$ of $\Omega$ by polygonal finite elements (or simply elements): each $\mathscr{T}^{h}$ consists of a finite number of elements, and each element $K \in \mathscr{T}^{h}$ is a bounded $m$-polygonal (open) domain (Fig.1), where $m$ is an integer $\geq 3$ and can differ with $K$. Thus the boundary $\partial K$ of $K \in \mathscr{T}^{h}$ is a closed simple polygonal curve composed of $m$ edges. We assume that $m$ is bounded from above by a positive integer $M(\geq 3)$, which is common to all the triangulations in $\left\{\mathscr{T}^{h}\right\}_{h>0}$. Notice here that non-convex elements are available for $m \geq 4$. We use the notation $e$ to denote an edge of $K$, which is assumed to be closed for convenience. The totality of edges of $K \in \mathscr{T}^{h}$ and $\mathscr{T}^{h}$ are denoted by $\mathscr{E}^{K}$ and $\mathscr{E}^{h}$, respectively

For theoretical treatment, we must impose some "regularity" conditions on the family $\left\{\mathscr{T}^{h}\right\}_{h>0}[9,11]$. We omit the details of regularity here, but, besides the trivial conditions such as $\cup_{K \in \mathscr{T} h} \bar{K}=\bar{\Omega}$ and $K \cap K^{\prime}=\emptyset$ for mutually different $K, K^{\prime} \in \mathscr{T}^{h}$, we require some additional conditions. In particular, each $K$ is not "too thin", and the intersection of closures of mutually different two elements $K, K^{\prime} \in \mathscr{T}^{h}$ is exclusively one of the following three sets : (i) empty set, (ii) one vertex, and (iii) one edge. Here we permit the flat interior angle to deal with the "hanging" nodes [9] (Fig. 2). ${ }^{1}$ The use of such nodes in DGFEM is effective to avoid the MPC (multi-point constraint) techniques employed in various exiting FEM codes [23, 29], and is also convenient in adaptive mesh refinements [9]. We will later try to present some additional regularity or geometrical conditions related to triangulations for DGFEM.

For each triangulation $\mathscr{T}^{h}$, we define its "skeleton" $\Gamma^{h}$ as the union of edges in $\mathscr{E}^{h}: \Gamma^{h}=\cup_{e \in \mathscr{E}_{h}} e$. For simplicity, we assume that the triangulations are so constructed that any edges intersecting with $\partial \Omega_{D}$ are entirely contained in $\partial \Omega_{D}$. In other words, such edges have no common points in $\partial \Omega \backslash \partial \Omega_{D}$.

The diameter and measure of $K$ are denoted by $h_{K}$ and $|K|$, respectively, while the length of an edge

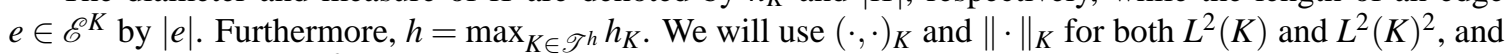
also define, for $\hat{u}, \hat{v} \in L^{2}(\partial K)$,

$$
\langle\hat{u}, \hat{v}\rangle_{\partial K}=\int_{\partial K} \hat{u} \hat{v} d s, \quad|\hat{v}|_{\partial K}=\langle\hat{v}, \hat{v}\rangle_{\partial K}^{1 / 2},
$$

where $d s$ is the infinitesimal line element on $\partial K$. Similarly, $\langle\cdot, \cdot\rangle_{e}$ and $|\cdot|_{e}$ are defined for each edge $e \in \mathscr{E}^{K}$. On the boundaries $\partial \Omega$ of $\Omega$ and $\partial K$ of $K$, the outward unit normal is well-defined almost everywhere, and is denoted by $n=\left\{n_{1}, n_{2}\right\}$.

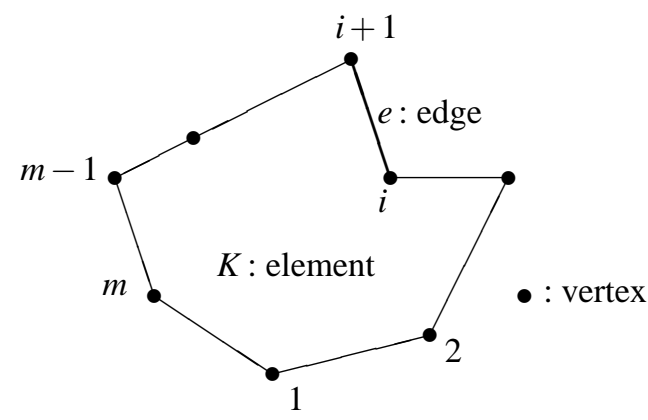

Figure 1: $m$-polygonal element $K$; non-convex case

\footnotetext{
${ }^{1}$ Strictly speaking, a vertex with the flat angle may not be a hanging node but rather a kind of degenerated one. Analysis of degenerated nodes in classical FEM is not necessarily easy [17, 32].
} 


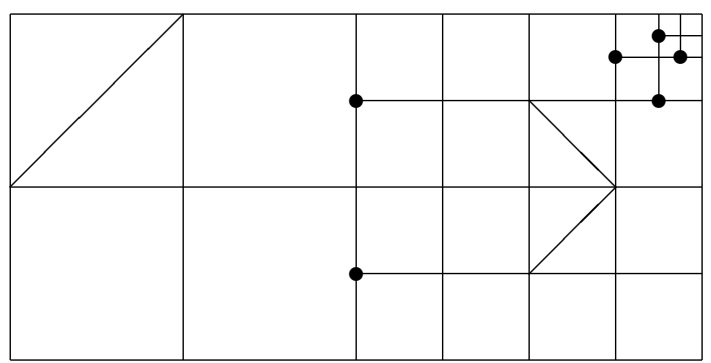

$\bullet$ : hanging node

Figure 2: Example of triangulation with hanging nodes

\subsection{Function spaces associated to triangulations}

Over $\mathscr{T}^{h}$, we consider the "broken" or piecewise Sobolev spaces $(\kappa>0)$ :

$$
H^{\kappa}\left(\mathscr{T}^{h}\right)=\left\{v \in L^{2}(\Omega) ;\left.v\right|_{K} \in H^{\kappa}(K)\left(\forall K \in \mathscr{T}^{h}\right)\right\},
$$

which can be identified with $\Pi_{K \in \mathscr{T} h} H^{\kappa}(K)$, where $H^{\kappa}(K)$ is the Sobolev space of (possibly fractional) order $\kappa$ over $K$. The norm and the standard semi-norm of this space are defined as usual and denoted by $\|\cdot\|_{\kappa, \mathscr{T}^{h}}$ and $|\cdot|_{\kappa, \mathscr{T} h}$, respectively. For $v \in H^{\frac{1}{2}+\sigma}\left(\mathscr{T}^{h}\right)(\sigma>0)$ and $K \in \mathscr{T}^{h}$, its trace to $\partial K$ is well defined as an element of $L^{2}(\partial K)$ and denoted by $\left.v\right|_{\partial K}$ or simply $v$, which can be double-valued on edges shared by two elements $[4,5,9]$. For $v \in H^{\frac{3}{2}+\sigma}\left(\mathscr{T}^{h}\right)(\sigma>0)$, we can define the trace of $\nabla v$ to $\partial K$ and the normal derivative $\partial v / \partial n$ there in the $L^{2}$ senses.

On $\Gamma^{h}$ of $\mathscr{T}^{h}$, we consider a kind of flux $\hat{v} \in L^{2}\left(\Gamma^{h}\right)$, which is single-valued on each edge shared by two elements, unlike various double-valued ones [4, 9]. To deal with the boundary condition in (1), define a subspace of $L^{2}\left(\Gamma^{h}\right)$ by

$$
L_{D}^{2}\left(\Gamma^{h}\right)=\left\{\hat{v} \in L^{2}\left(\Gamma^{h}\right) ; \hat{v}=0 \text { on } \partial \Omega_{D}\right\} .
$$

For each $\left\{\mathscr{T}^{h}\right\}_{h>0}$, let us define some (semi-)norms for arguments $\{v, \hat{v}\}$, cf. [9]:

$$
\begin{gathered}
\|v\|_{h}^{2}=\|v\|_{\Omega}^{2}+\sum_{K \in \mathscr{T}^{h}} \sum_{e \in \mathscr{E}^{K}}|e| \cdot|v|_{e}^{2} ; \forall v \in H^{\frac{1}{2}+\sigma}\left(\mathscr{T}^{h}\right), \\
|\{v, \hat{v}\}|_{h}^{2}=\left\|\nabla_{h} v\right\|_{\Omega}^{2}+\sum_{K \in \mathscr{T}^{h}} \sum_{e \in \mathscr{E}^{K}} \frac{1}{|e|}|v-\hat{v}|_{e}^{2} ; \forall\{v, \hat{v}\} \in H^{1}\left(\mathscr{T}^{h}\right) \times L^{2}\left(\Gamma^{h}\right), \\
\|\{v, \hat{v}\}\|_{h}^{2}=|\{v, \hat{v}\}|_{h}^{2}+\sum_{K \in \mathscr{T}^{h}} \sum_{e \in \mathscr{E}^{K}}|e| \cdot|\nabla v|_{e}^{2} ; \forall\{v, \hat{v}\} \in H^{\frac{3}{2}+\sigma}\left(\mathscr{T}^{h}\right) \times L^{2}\left(\Gamma^{h}\right),
\end{gathered}
$$

where $\sigma>0, v$ on $e$ or $\left.\left(\left.v\right|_{K}\right)\right|_{e}$ implies the trace of $\left.v\right|_{K}$ to $e \in \mathscr{E}^{h}, \nabla v$ on $e$ or $\left.\nabla\left(\left.v\right|_{K}\right)\right|_{e}$ does the trace of $\nabla\left(\left.v\right|_{K}\right)$ to $e$, and $\nabla_{h}: H^{1}\left(\mathscr{T}^{h}\right) \rightarrow L^{2}(\Omega)^{2}$ is characterized by $\left.\left(\nabla_{h} v\right)\right|_{K}=\nabla\left(\left.v\right|_{K}\right)$ for $v \in H^{1}\left(\mathscr{T}^{h}\right)$ and $K \in \mathscr{T}^{h}$. Notice here that $v$ and $\nabla v$ can be double-valued on $e$ but $\hat{v}$ is not so. All of these (semi-)norms are mesh-dependent. The first one is a norm, while the other two are semi-norms in general but become norms if $\partial \Omega_{D}$ has positive total length, cf. [4].

\subsection{Lifting operators}

To consider the local lifting operator [4] for each $K \in \mathscr{T}^{h}$, let us introduce

$$
\begin{array}{r}
Q^{K}=\text { finite dimensional subspace of } L^{2}(K), \text { such as the space } \\
P_{k}(K) \text { of polynomials on } K \text { of degree } \leq k(k=0,1,2, . .) .
\end{array}
$$


Then, the local lifting operator $R_{K}: g \in L^{2}(\partial K) \mapsto p \in\left(Q^{K}\right)^{2}$ is well-defined as : given $g \in L^{2}(\partial K)$, find $p=\left\{p_{1}, p_{2}\right\} \in\left(Q^{K}\right)^{2}$ such that

$$
(p, q)_{K}=\langle g, q \cdot n\rangle_{\partial K} ; \forall q=\left\{q_{1}, q_{2}\right\} \in\left(Q^{K}\right)^{2},
$$

where $q \cdot n=q_{1} n_{1}+q_{2} n_{2}$, and the minus sign is sometimes added to the right-hand side, cf. e.g. [4]. Identifying $Q^{h}:=\Pi_{K \in \mathscr{T}_{h}} Q^{K}$ with a subspace of $L^{2}(\Omega)$ and making the identification $\Pi_{K \in \mathscr{T}^{h}}\left(Q^{K}\right)^{2}=\left(Q^{h}\right)^{2}$, the global lifting operator is defined by

$$
R_{h}: \tilde{g}=\left\{g_{\partial K}\right\}_{K \in \mathscr{T}^{h}} \in \Pi_{K \in \mathscr{T}^{h}} L^{2}(\partial K) \mapsto\left\{R_{K} g_{\partial K}\right\}_{K \in \mathscr{T}^{h}} \in\left(Q^{h}\right)^{2} \subset L^{2}(\Omega)^{2} .
$$

Since $\hat{v} \in L^{2}\left(\Gamma^{h}\right)$ is single-valued on every edge $e \in \mathscr{E}^{h}$, it can be naturally identified with an element

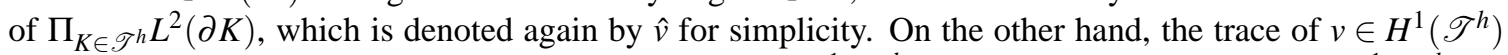
to $e$ may be double-valued if $e \not \subset \partial \Omega$. To use $R_{h}$ for $v \in H^{1}\left(\mathscr{T}^{h}\right)$, let us define an operator $S_{h}: H^{1}\left(\mathscr{T}^{h}\right) \rightarrow$ $\Pi_{K \in \mathscr{T}_{h}} L^{2}(\partial K)$ by

$$
S_{h} v=\left\{\left.\left(\left.v\right|_{K}\right)\right|_{\partial K}\right\}_{K \in \mathscr{T}^{h}}
$$

We can now operate $R_{h}$ on $S_{h} v$ for $v \in H^{1}\left(\mathscr{T}^{h}\right)$ to find $R_{h} S_{h} v \in\left(Q^{h}\right)^{2} \subset L^{2}(\Omega)^{2}$.

\subsection{Finite element spaces}

To approximate $\{v, \hat{v}\} \in H^{\frac{3}{2}+\sigma}\left(\mathscr{T}^{h}\right) \times L^{2}\left(\Gamma^{h}\right)\left(0<\sigma \leq \frac{1}{2}\right)$ associated to $\mathscr{T}^{h}$, let us prepare two finite dimensional spaces :

$$
\begin{aligned}
& U^{h}=\text { finite dimensional subspace of } H^{\frac{3}{2}+\sigma}\left(\mathscr{T}^{h}\right)\left(0<\sigma \leq \frac{1}{2}\right), \\
& \hat{U}^{h}=\text { finite dimensional subspace of } L^{2}\left(\Gamma^{h}\right) \text { or } C\left(\Gamma^{h}\right),
\end{aligned}
$$

where $C\left(\Gamma^{h}\right)$ denotes the space of continuous functions on $\Gamma^{h}$. Examples of $U^{h}$ are $\Pi_{K \in T_{h}} P_{k}(K)(k \in N)$, while those of $\hat{U}^{h}$ are $\Pi_{e \in \mathscr{E} h} P_{k}(e)(k \in N)$ or their subsets in $C\left(\Gamma^{h}\right)$, where $P_{k}(e)$ is the space of polynomials on $e$ of degree $\leq k$. In the present setting, $\hat{v}_{h} \in \hat{U}^{h}$ is not double-valued on each edge $e$. Moreover, to deal with the homogeneous Dirichlet condition in (1), let us introduce the subspace of $\hat{U}^{h}$ by

$$
\hat{U}_{D}^{h}=\left\{\hat{v}_{h} \in \hat{U}^{h} ; \hat{v}_{h}=0 \text { on } \partial \Omega_{D}\right\}
$$

From the assumptions on $\Gamma^{h}$, the above condition on $\hat{v}_{h}$ is equivalent to: $\hat{v}_{h}$ vanishes completely on every edge contained in $\Gamma^{h} \cap \partial \Omega_{D}$.

Then the finite element spaces are given by

$$
V^{h}=U^{h} \times \hat{U}^{h}, \quad V_{D}^{h}=U^{h} \times \hat{U}_{D}^{h} .
$$

Under appropriate conditions on $\left\{\mathscr{T}^{h}\right\}_{h>0}$ and $V^{h}$, we can show $\||\cdot|\|_{h}$ is equivalent to $|\cdot|_{h}$ over $V^{h}$, cf. [4]. We also need $Q^{K}$ for each $K \in \mathscr{T}^{h}$ to define $Q^{h}$ and use $R_{h}$.

\subsection{Bilinear forms}

Let us consider two symmetric bilinear forms associated to $-\Delta[26,27]$ :

$$
\begin{gathered}
B_{h}(\{u, \hat{u}\},\{v, \hat{v}\})=\left(\nabla_{h} u, \nabla_{h} v\right)_{\Omega}+\sum_{K \in \mathscr{T}^{h}}\left(\left\langle\frac{\partial u}{\partial n}, \hat{v}-v\right\rangle_{\partial K}+\left\langle\frac{\partial v}{\partial n}, \hat{u}-u\right\rangle_{\partial K}\right) \\
+\sum_{K \in \mathscr{T}^{h}} \sum_{e \in \mathscr{E}^{K}} \frac{\eta_{0}}{|e|}\langle\hat{u}-u, \hat{v}-v\rangle_{e}\left[+\left(R_{h}\left(\hat{u}-S_{h} u\right), R_{h}\left(\hat{v}-S_{h} v\right)\right)_{\Omega}\right] \\
\forall\{u, \hat{u}\},\{v, \hat{v}\} \in H^{\frac{3}{2}+\sigma}\left(\mathscr{T}^{h}\right) \times L^{2}\left(\Gamma^{h}\right)\left(0<\sigma \leq \frac{1}{2}\right),
\end{gathered}
$$


where $\frac{\partial u}{\partial n}=(\nabla u) \cdot n, \frac{\partial v}{\partial n}=(\nabla v) \cdot n$, and $\eta_{0}>0$ is the interior penalty parameter. We have now introduced two bilinear forms, whose difference lies in the use or non-use of the term in $[\cdot]$. We can also consider other bilinear forms including non-symmetric ones [4], but we here restrict our analysis to the above two.

If $V^{h}$ and $Q^{K}$ are so chosen that $\nabla\left(\left.v_{h}\right|_{K}\right) \in Q^{K}$ for all $v_{h} \in U^{h}$ and $K \in \mathscr{T}^{h}$, we have from (11) that $\left(R_{K}\left(\hat{v}_{h}-v_{h}\right), \nabla u_{h}\right)_{K}=\left\langle\hat{v}_{h}-v_{h}, \frac{\partial u_{h}}{\partial n}\right\rangle_{\partial K}$ for all $u_{h} \in U^{h}$. In such a case, it holds that, for all $\left\{u_{h}, \hat{u}_{h}\right\},\left\{v_{h}, \hat{v}_{h}\right\} \in$ $V^{h}$

$$
\begin{aligned}
& B_{h}\left(\left\{u_{h}, \hat{u}_{h}\right\},\left\{v_{h}, \hat{v}_{h}\right\}\right)=\left(\nabla_{h} u_{h}+R_{h}\left(\hat{u}_{h}-S_{h} u_{h}\right), \nabla_{h} v_{h}+R_{h}\left(\hat{v}_{h}-S_{h} v_{h}\right)\right)_{\Omega} \\
& +\sum_{K \in \mathscr{T} h} \sum_{e \in \mathscr{E}_{K}} \frac{\eta_{0}}{|e|}\left\langle\hat{u}_{h}-u_{h}, \hat{v}_{h}-v_{h}\right\rangle_{e}\left[-\left(R_{h}\left(\hat{u}_{h}-S_{h} u_{h}\right), R_{h}\left(\hat{v}_{h}-S_{h} v_{h}\right)_{\Omega}\right],\right.
\end{aligned}
$$

where the last term in $[\cdot]$ is absent when the last term in (18) is active. We can see from (19) that the last term in (18) is effective to increase the positivity of the bilinear form when used in finite element schemes.

Bilinear forms like above $B_{h}$ 's are used to solve numerically the Poisson equation when $\partial \Omega_{D} \neq \emptyset$ : given $f \in L^{2}(\Omega)$, find $\left\{u_{h} \cdot \hat{u}_{h}\right\} \in V_{D}^{h}$ that satisfies

$$
B_{h}\left(\left\{u_{h}, \hat{u}_{h}\right\},\left\{v_{h}, \hat{v}_{h}\right\}\right)=\left(f, v_{h}\right)_{\Omega} ; \forall\left\{v_{h}, \hat{v}_{h}\right\} \in V_{D}^{h} .
$$

When we deal with (2) numerically, we need the term $\left(u_{h}, v_{h}\right)_{\Omega}$ on the left-hand side.

\subsection{Comments on other symmetric DGFEM}

In the two bilinear forms above, $\hat{v}$ is independent of $v$. Introducing appropriate constraints between them, we can obtain some genuine (non-hybridized) DGFEM. To this end, define $\{\{v\}\} \in L^{2}\left(\Gamma^{h}\right)$ for $v \in H^{1}\left(\mathscr{T}^{h}\right)$ as : for an edge $e \in \mathscr{E}^{h}$, we set $\left.\{\{v\}\}\right|_{e}=\left.v\right|_{e}$ if $e \subset \partial \Omega$, while we set as follows if $e$ is shared by two elements $K_{1}, K_{2} \in \mathscr{T}^{h}$;

$$
\left.\{\{v\}\}\right|_{e}=\frac{1}{2}\left(v_{1}+v_{2}\right) \quad(\text { simple averaging })
$$

where $v_{1}$ ( $v_{2}$ resp.) is the trace of $v \mid K_{1}\left(v \mid K_{2}\right.$ resp.) to $e$.

Using such $\{\{v\}\}$ as $\hat{v}$ when $e \not \subset \partial \Omega_{D}$ in our bilinear forms, we have IP (Interior Penalty) method and a kind of LDG (Local Discontinuous Galerkin) one $[4,9,16]$. The difference is that the lifting term in (18) is employed in LDG but not in IP. Such modification reduces the number of unknowns for the linear simultaneous equations associated to (20), but the sparseness of the coefficient matrices may deteriorate [22].

\section{Main Results}

In this section, we will show a discrete analog of the well-known Rellich theorem for the considered DGFEM.

Along with the conditions in Sec.2, we make some additional assumptions, which can be actually proved under appropriate settings on the family of triangulations and finite element spaces. For the moment, however, we postpone such technical processes and prove our main results under the hypotheses below, which are common in analysis of DGFEM [4, 9, 27] but slightly modified for our purposes. Clearly, the results hold true for various DGFEM other than those in the preceding sections, so long as they satisfy such conditions.

[H0] Approximation capability For any $u \in H^{\frac{3}{2}+\sigma}(\Omega)$ and $p \in H^{\frac{1}{2}+\sigma}(\Omega)\left(0<\sigma \leq \frac{1}{2}\right)$, there exists a family $\left\{\left\{u_{h}^{*}, \hat{u}_{h}^{*}, p_{h}^{*}\right\} \in V^{h} \times Q^{h}\right\}_{h>0}$ such that

$$
\left\|\left\{u-u_{h}^{*}, \hat{u}-\hat{u}_{h}^{*}\right\}\right\|_{h}+\left\|p-p_{h}^{*}\right\|_{h} \leq C_{a} h^{\frac{1}{2}+\sigma}\left(\|u\|_{\frac{3}{2}+\sigma, \Omega}+\|p\|_{\frac{1}{2}+\sigma, \Omega}\right),
$$

where $C_{a}$ is a generic positive constant independent of $u, p$ and $h>0$, and $\hat{u}$ denotes the trace of $u$ to $\Gamma^{h}$, i.e., $\hat{u}=\left.u\right|_{\Gamma^{h}}$. Moreover, when $u$ belongs to $H_{D}^{1}(\Omega) \cap H^{\frac{3}{2}+\sigma}(\Omega)$, the same estimate holds with each $\left\{u_{h}^{*}, \hat{u}_{h}^{*}\right\}$ chosen from $V_{D}^{h}$. 
[H1] Consistency Let $u \in H_{D}^{1}(\Omega)$ be the solution of (2) for arbitrarily given $f \in L^{2}(\Omega)$. Then it holds that, with $\hat{u}=\left.u\right|_{\Gamma^{h}}$, and for all $h>0$ and $\left\{v_{h}, \hat{v}_{h}\right\} \in V_{D}^{h}$,

$$
B_{h}\left(\{u, \hat{u}\},\left\{v_{h}, \hat{v}_{h}\right\}\right)+\left(u, v_{h}\right)_{\Omega}=\left(f, v_{h}\right)_{\Omega} .
$$

[H2] Boundedness There exists a positive constant $C_{b}$ such that, for all $h>0$ and $\{u, \hat{u}\},\{v, \hat{v}\} \in$ $H^{\frac{3}{2}+\sigma}\left(\mathscr{T}^{h}\right) \times L^{2}\left(\Gamma^{h}\right)\left(0<\sigma \leq \frac{1}{2}\right)$,

$$
\left|B_{h}(\{u, \hat{u}\},\{v, \hat{v}\})\right| \leq C_{b} \|\left\{\{u, \hat{u}\}\left\|_{h} \mid\right\|\{v, \hat{v}\} \|_{h} .\right.
$$

[H3] Stability (Coerciveness) There exists a positive constant $C_{s}$ such that, for all $h>0$ and $\left\{v_{h}, \hat{v}_{h}\right\} \in V^{h}$,

$$
\left|B_{h}\left(\left\{v_{h}, \hat{v}_{h}\right\},\left\{v_{h}, \hat{v}_{h}\right\}\right)\right| \geq C_{s}\left|\left\{v_{h}, \hat{v}_{h}\right\}\right|_{h}^{2} .
$$

[H4] Assumptions on $Q^{K}$ and $R_{K} \quad$ For all $h>0, K \in \mathscr{T}^{h}$ and $v_{h} \in U^{h}$, it holds that $\nabla\left(\left.v_{h}\right|_{K}\right) \in Q^{K}$, so that (19) is available. Moreover, there exists a positive constant $C_{r}$ such that, for all $h>0, K \in \mathscr{T}^{h}$ and $g \in L^{2}(\partial K)$

$$
\left\|R_{K} g\right\|_{K} \leq C_{r}\left(\left.\sum_{e \in \mathscr{E}^{K}} \frac{1}{|e|}|g|_{e}\right|_{e} ^{2}\right)^{\frac{1}{2}},
$$

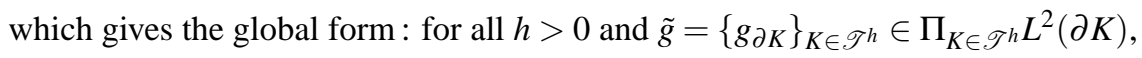

$$
\left\|R_{h} \tilde{g}\right\|_{\Omega} \leq C_{r}\left(\left.\sum_{K \in \mathscr{T}^{h}} \sum_{e \in \mathscr{E}^{K}} \frac{1}{|e|}\left|g_{\partial K}\right| e\right|_{e} ^{2}\right)^{\frac{1}{2}} .
$$

Remark 1. For the present purposes, (22) of [H0] can be much weakened: for example, the exponent $\frac{1}{2}+\sigma$ there can be replaced with arbitrarily small positive constants. Consistency condition [H1] is that for (2) but not for the Poisson equation. Under [H1], the adjoint consistency [4] holds automatically for the present types of symmetric formulations, and is effective to apply the Aubin-Nitsche trick [11]. Moreover, the magnitude of interior penalty parameter $\eta_{0}$ must be large enough for [H3] to hold for the bilinear form $B_{h}$ in (18) without the lifting term [4, 9, 27].

Theorem 1. Let $\left\{\left\{u_{h}, \hat{u}_{h}\right\} \in V_{D}^{h}\right\}_{h>0}$ be a family associated to $\left\{\mathscr{T}^{h}\right\}_{h>0}$ such that $\left|\left\{u_{h}, \hat{u}_{h}\right\}\right|_{h}^{2}+\left\|u_{h}\right\|_{\Omega}^{2} \leq 1$. We assume [H0] through [H4] besides the conditions in Sec.2. Then there exist a function $u_{0} \in H_{D}^{1}(\Omega)$ and a subfamily, denoted again by $\left\{\left\{u_{h}, \hat{u}_{h}\right\}\right\}_{h>0}$ for convenience, such that, as $h \downarrow 0$,

$$
\begin{aligned}
& u_{h} \rightarrow u_{0} \text { strongly in } L^{2}(\Omega),\left.\left.u_{h}\right|_{\partial \Omega_{D}} \rightarrow u_{0}\right|_{\partial \Omega_{D}}=0 \text { strongly in } L^{2}\left(\partial \Omega_{D}\right), \\
& \nabla_{h} u_{h}+R_{h}\left(\hat{u}_{h}-S_{h} u_{h}\right) \rightarrow \nabla u_{0} \text { weakly in } L^{2}(\Omega)^{2} .
\end{aligned}
$$

Remark 2. As may be seen from the proof of Lemma 1 below, the expression $\nabla_{h} u_{h}+R_{h}\left(\hat{u}_{h}-S_{h} u_{h}\right)$ is a natural approximation to the distributional derivative of $u_{h}$. Weak convergence of $\left\{\nabla_{h} u_{h}\right\}_{h>0}$ to $\nabla u_{0}$ is not shown here. To assure such convergence, we may need modifications of the interior penalty terms.

Proof. For the specified family $\left\{\left\{u_{h}, \hat{u}_{h}\right\} \in V_{D}^{h}\right\}_{h>0}$, define $\left\{u^{h} \in H_{D}^{1}(\Omega)\right\}_{h>0}$ and $\left\{\left\{u_{h}^{h}, \hat{u}_{h}^{h}\right\} \in V_{D}^{h}\right\}_{h>0}$ by, for each $\mathscr{T}^{h}$,

$$
\begin{gathered}
\left(\nabla u^{h}, \nabla v\right)_{\Omega}+\left(u^{h}, v\right)_{\Omega}=\left(u_{h}, v\right)_{\Omega} ; \forall v \in H_{D}^{1}(\Omega), \\
B_{h}\left(\left\{u_{h}^{h}, \hat{u}_{h}^{h}\right\},\left\{v_{h}, \hat{v}_{h}\right\}\right)+\left(u_{h}^{h}, v_{h}\right)_{\Omega}=\left(u_{h}, v_{h}\right)_{\Omega} ; \forall\left\{v_{h}, \hat{v}_{h}\right\} \in V_{D}^{h} .
\end{gathered}
$$

We will now prove in several steps.

$\mathbf{1}^{\circ}$ Equating $v$ to $u^{h}$ in $(a)$ and using the Schwarz inequality, we find $\left\|u^{h}\right\|_{1, \Omega} \leq\left\|u_{h}\right\|_{\Omega} \leq 1$. Applying the Rellich theorem and standard arguments to the families $\left\{u_{h}\right\}_{h>0}$ and $\left\{u^{h}\right\}_{h>0}$, we can choose their subfamilies, denoted by the same notations, with their limit functions $u_{0} \in L^{2}(\Omega)$ and $u^{0} \in H_{D}^{1}(\Omega)$ such that, as $h \downarrow 0$,

$$
u_{h} \rightarrow u_{0} \text { weakly in } L^{2}(\Omega)
$$


Then, taking the limit in $(a)$ for the subfamilies, we find that $u^{0}$ and $u_{0}$ satisfy

$$
\left(\nabla u^{0}, \nabla v\right)_{\Omega}+\left(u^{0}, v\right)_{\Omega}=\left(u_{0}, v\right)_{\Omega} ; \forall v \in H_{D}^{1}(\Omega),
$$

from which we have $\left(u_{0}, u^{0}\right)_{\Omega}=\left\|\nabla u^{0}\right\|_{\Omega}^{2}+\left\|u^{0}\right\|_{\Omega}^{2}=\left\|u^{0}\right\|_{1, \Omega}^{2}$. Similarly, we obtain from $(a)$ that $\left(u_{h}, u^{h}\right)_{\Omega}=$ $\left\|u^{h}\right\|_{1, \Omega}^{2}$. Since $\left(u_{h}, u^{h}\right)_{\Omega} \rightarrow\left(u_{0}, u^{0}\right)_{\Omega}(h \downarrow 0)$ by $(i)$ and $(i i)$, we can see $\left\|u^{h}\right\|_{1, \Omega} \rightarrow\left\|u^{0}\right\|_{1, \Omega}$, so that

$$
u^{h} \rightarrow u^{0} \text { strongly in } H_{D}^{1}(\Omega) .
$$

$2^{\circ}$ Since $u_{h} \in L^{2}(\Omega), u^{h}$ of $(a)$ belongs to $H_{D}^{1}(\Omega) \cap H^{\frac{2}{3}+\sigma}(\Omega)$. Then we can perform error analysis of $\left\{\left\{u_{h}^{h}, \hat{u}_{h}^{h}\right\}\right\}_{h>0}$ with respect to $u^{h}$ using the hypotheses from [H0] through [H4] together with (2) as in [26, 27], and we have

$$
\left\|\left\{u^{h}-u_{h}^{h}, \hat{u}^{h}-\hat{u}_{h}^{h}\right\}\right\|_{h} \leq C h^{\frac{1}{2}+\sigma}\left\|u^{h}\right\|_{\frac{3}{2}+\sigma, \Omega} \leq C_{D} C h^{\frac{1}{2}+\sigma}\left\|u_{h}\right\|_{\Omega}
$$

where $\hat{u}^{h}$ is the trace of $u^{h}$ to $\Gamma^{h}$ (which is not double-valued), $C_{D}$ is the constant in (2), and $C$ is a positive constant dependent only on $C_{a}, C_{b}$ and $C_{s}{ }^{2}$

$\mathbf{3}^{\circ}$ By [H1], we have

$$
B_{h}\left(\left\{u^{h}, \hat{u}^{h}\right\},\left\{u_{h}, \hat{u}_{h}\right\}\right)+\left(u^{h}, v_{h}\right)_{\Omega}=\left(u_{h}, u_{h}\right)_{\Omega},
$$

which can be concretely rewritten by

$$
\left(\nabla u^{h}, \nabla_{h} u_{h}\right)_{\Omega}+\sum_{K \in \mathscr{T}^{h}}\left\langle\frac{\partial u^{h}}{\partial n}, \hat{u}_{h}-u_{h}\right\rangle_{\partial K}+\left(u^{h}, u_{h}\right)_{\Omega}=\left(u_{h}, u_{h}\right)_{\Omega} .
$$

Here, terms including $\hat{u}^{h}-\hat{u}$ in the original expression (18) for $B_{h}\left(\left\{u^{h}, \hat{u}^{h}\right\},\left\{u_{h}, \hat{u}_{h}\right\}\right)$ have vanished thanks to $\hat{u}^{h}=\left.u^{h}\right|_{\Gamma^{h}}$. By [H4] and using $u_{h}^{h} \in U^{h}$ of $(b)$ in the second term of the above, we find $\sum_{K \in \mathscr{T}^{h}}\left\langle\frac{\partial u^{h}}{\partial n}, \hat{u}_{h}-\right.$ $\left.u_{h}\right\rangle_{\partial K}=\sum_{K \in \mathscr{T} h}\left\langle\frac{\partial\left(u^{h}-u_{h}^{h}\right)}{\partial n}, \hat{u}_{h}-u_{h}\right\rangle_{\partial K}+\left(\nabla_{h} u_{h}^{h}, R_{h}\left(\hat{u}_{h}-S_{h} u_{h}\right)\right)_{\Omega}$, so that the above equality is further rewritten as

$$
\begin{aligned}
& \left(\nabla_{h} u_{h}^{h}, \nabla_{h} u_{h}+R_{h}\left(\hat{u}_{h}-S_{h} u_{h}\right)\right)_{\Omega}+\left(\nabla u^{h}-\nabla_{h} u_{h}^{h}, \nabla_{h} u_{h}\right)_{\Omega} \\
& +\sum_{K \in \mathscr{T} h}\left\langle\frac{\partial\left(u^{h}-u_{h}^{h}\right)}{\partial n}, \hat{u}_{h}-u_{h}\right\rangle_{\partial K}+\left(u^{h}, u_{h}\right)_{\Omega}=\left(u_{h}, u_{h}\right)_{\Omega} .
\end{aligned}
$$

$4^{\circ}$ As regards $\nabla_{h} u_{h}+R_{h}\left(\hat{u}_{h}-S_{h} u_{h}\right)$ in $(e)$, we can show the following lemma.

Lemma 1. Let $\left\{\left\{u_{h}, \hat{u}_{h}\right\} \in V_{D}^{h}\right\}_{h>0}$ be the subfamily which is selected to satisfy $(i)$. Then, $u_{0} \in L^{2}(\Omega)$ in $(i)$ also belongs to $H_{D}^{1}(\Omega)$, and, as $h \downarrow 0$,

$$
\nabla_{h} u_{h}+R_{h}\left(\hat{u}_{h}-S_{h} u_{h}\right) \rightarrow \nabla u_{0} \text { weakly in } L^{2}(\Omega)^{2} .
$$

We will give the proof of Lemma 1 later. Once we admit this lemma, we find with the aid of (ii), (iii) and $(d)$ that, as $h \downarrow 0$,

$$
\begin{aligned}
\left(\nabla_{h} u_{h}^{h}, \nabla_{h} u_{h}+R_{h}\left(\hat{u}_{h}-S_{h} u_{h}\right)\right)_{\Omega} & =\left(\nabla_{h} u_{h}^{h}-\nabla u^{h}+\nabla u^{h}, \nabla_{h} u_{h}+R_{h}\left(\hat{u}_{h}-S_{h} u_{h}\right)\right)_{\Omega} \\
& \rightarrow\left(\nabla u^{0}, \nabla u_{0}\right)_{\Omega} .
\end{aligned}
$$

Similarly, using $(i),(i i),(d)$ and $\left\|\nabla_{h} u_{h}\right\|_{\Omega} \leq 1$, we can also obtain that, as $h \downarrow 0$,

$$
\left(\nabla u^{h}-\nabla_{h} u_{h}^{h}, \nabla_{h} u_{h}\right)_{\Omega} \rightarrow 0, \quad\left(u^{h}, u_{h}\right)_{\Omega} \rightarrow\left(u^{0}, u_{0}\right)_{\Omega} .
$$

Moreover, we have

$$
\sum_{K \in \mathscr{T}^{h}}\left\langle\frac{\partial\left(u^{h}-u_{h}^{h}\right)}{\partial n}, \hat{u}_{h}-u_{h}\right\rangle_{\partial K} \rightarrow 0 \quad(h \downarrow 0),
$$

${ }^{2}$ Notations $C, C_{1}, C_{2}$ etc. will be used as various generic positive constants. 
since

$$
\begin{aligned}
& \left|\sum_{K \in \mathscr{T}^{h}}\left\langle\frac{\partial\left(u^{h}-u_{h}^{h}\right)}{\partial n}, \hat{u}_{h}-u_{h}\right\rangle_{\partial K}\right| \leq \sum_{K \in \mathscr{T}^{h}} \sum_{e \in \mathscr{E}^{K}}\left|\nabla\left(u^{h}-u_{h}^{h}\right)\right|_{e} \cdot\left|\hat{u}_{h}-u_{h}\right|_{e} \\
& \leq\left[\sum_{K \in \mathscr{T}^{h}} \sum_{e \in \mathscr{E}^{K}}|e| \cdot\left|\nabla\left(u^{h}-u_{h}^{h}\right)\right|_{e}^{2}\right]^{1 / 2}\left[\sum_{K \in \mathscr{T}^{h}} \sum_{e \in \mathscr{C}^{K}} \frac{1}{|e|}\left|\hat{u}_{h}-u_{h}\right|_{e}^{2}\right]^{1 / 2} \\
& \leq\left\|\left|\left\|\left\{u^{h}-u_{h}^{h}, \hat{u}^{h}-\hat{u}_{h}^{h}\right\}\right\|\left\|_{h} \cdot\left|\left\{u_{h}, \hat{u}_{h}\right\}\right|_{h} \leq \mid\right\|\left\{u^{h}-u_{h}^{h}, \hat{u}^{h}-\hat{u}_{h}^{h}\right\} \|_{h} .\right.\right.
\end{aligned}
$$

From these results, the left-hand side of $(e)$ converges to $\left(\nabla u^{0}, \nabla u_{0}\right)_{\Omega}+\left(u^{0}, u_{0}\right)_{\Omega}$, which is equal to $\left(u_{0}, u_{0}\right)_{\Omega}$ by $(c)$. In view of $(e)$, this implies

$$
\left(u_{h}, u_{h}\right)_{\Omega} \rightarrow\left(u_{0}, u_{0}\right)_{\Omega} \quad(h \downarrow 0),
$$

which together with $(i)$ assures that the former part of (28) holds true.

$5^{\circ}$ Let us prove that $\left\{\left.u_{h}\right|_{\partial \Omega_{D}}\right\}_{h>0}$ converges strongly to 0 in $L^{2}\left(\partial \Omega_{D}\right)$ as $h \downarrow 0$. But this follows from the estimation

$$
\int_{\partial \Omega_{D}}\left|u_{h}\right|^{2} d s=\int_{\partial \Omega_{D}}\left|u_{h}-\hat{u}_{h}\right|^{2} d s \leq \sum_{K \in \mathscr{T} h} \sum_{e \in \mathscr{E}^{K}}|e|^{2} \frac{1}{|e|}\left|u_{h}-\hat{u}_{h}\right|_{e}^{2} \leq h^{2}\left|\left\{u_{h}, \hat{u}_{h}\right\}\right|_{h}^{2} \leq h^{2} .
$$

$6^{\circ}$ Finally, let us prove Lemma 1 . To this end, we use the vector-valued test function $\varphi=\left\{\varphi_{1}, \varphi_{2}\right\} \in C_{0}^{\infty}(\Omega)^{2}$ to consider distributional derivatives of $u_{h} \in U^{h}$. Then the value of $\nabla u_{h}$ at $\varphi$ is given by $-\left(u_{h} \text {, } \operatorname{div} \varphi\right)_{\Omega}$, which is rewritten as

$$
-\left(u_{h}, \operatorname{div} \varphi\right)_{\Omega}=\left(\nabla_{h} u_{h}, \varphi\right)_{\Omega}+\sum_{K \in \mathscr{T}^{h}} \int_{\partial K}\left(\hat{u}_{h}-u_{h}\right)(\varphi \cdot n) d s,
$$

where the Green formula is used with the inter-element continuity of $\varphi$ taken into account, and $\hat{u}_{h} \in \hat{U}_{D}^{h}$ is inserted for later use. The use of such $\hat{u}_{h}$ is justified since $\hat{u}_{h}$ is single-valued on $\Gamma^{h}$ and $\left.\varphi\right|_{\partial \Omega}=0$.

For each $\left.\varphi \in C_{0}^{\infty}(\Omega)^{2} \subset H^{\frac{1}{2}+\sigma}(\Omega)\right\}^{2}\left(0<\sigma \leq \frac{1}{2}\right)$, we can choose by [H0] a family of functions $\left\{\varphi_{h}=\right.$ $\left.\left\{\varphi_{h 1}, \varphi_{h 2}\right\} \in\left(Q^{h}\right)^{2}\right\}_{h>0}$ such that

$$
\sum_{i=1}^{2}\left\|\varphi_{i}-\varphi_{h i}\right\|_{h} \rightarrow 0 \quad(h \downarrow 0) .
$$

Using such $\varphi_{h}$, we have

$$
\begin{gathered}
-\left(u_{h}, \operatorname{div} \varphi\right)_{\Omega}=\left(\nabla_{h} u_{h}, \varphi\right)_{\Omega}+\sum_{K \in \mathscr{T} h} \int_{\partial K}\left(\hat{u}_{h}-u_{h}\right)\left[\left(\varphi-\varphi_{h}+\varphi_{h}\right) \cdot n\right] d s \\
=\left(\nabla_{h} u_{h}, \varphi\right)_{\Omega}+\sum_{K \in \mathscr{T} h} \int_{\partial K}\left(\hat{u}_{h}-u_{h}\right)\left[\left(\varphi-\varphi_{h}\right) \cdot n\right] d s+\left(R_{h}\left(\hat{u}_{h}-S_{h} u_{h}\right), \varphi_{h}\right)_{\Omega} \\
=\left(\nabla_{h} u_{h}+R_{h}\left(\hat{u}_{h}-S_{h} u_{h}\right), \varphi_{h}\right)_{\Omega}+\left(\nabla_{h} u_{h}, \varphi-\varphi_{h}\right)_{\Omega} \\
\quad+\sum_{K \in \mathscr{T} h} \int_{\partial K}\left(\hat{u}_{h}-u_{h}\right)\left[\left(\varphi-\varphi_{h}\right) \cdot n\right] d s
\end{gathered}
$$

It is easy to see that the last two terms converge to 0 as $h \downarrow 0$, since

$$
\begin{aligned}
& \left|\left(\nabla_{h} u_{h}, \varphi-\varphi_{h}\right)_{\Omega}\right| \leq\left\|\nabla_{h} u_{h}\right\|_{\Omega} \cdot\left\|\varphi-\varphi_{h}\right\|_{\Omega} \rightarrow 0 \quad\left(\text { by }(g) \text { and }\left\|\nabla_{h} u_{h}\right\|_{\Omega} \leq 1\right), \\
& \left|\sum_{K \in \mathscr{T}^{h}} \int_{\partial K}\left(\hat{u}_{h}-u_{h}\right)\left[\left(\varphi-\varphi_{h}\right) \cdot n\right] d s\right| \\
& \leq\left[\sum_{K \in \mathscr{T}^{h}} \sum_{e \in \mathscr{E}^{K}} \frac{1}{|e|}\left|\hat{u}_{h}-u_{h}\right|_{e}^{2}\right]^{1 / 2}\left[\sum_{K \in \mathscr{T}^{h}} \sum_{e \in \mathscr{E}^{K}}|e| \sum_{i=1}^{2}\left|\varphi_{i}-\varphi_{h i}\right|_{e}^{2}\right]^{1 / 2} \\
& \leq\left|\left\|\left\{u^{h}-u_{h}^{h}, \hat{u}^{h}-\hat{u}_{h}^{h}\right\} \mid\right\|_{h} \sum_{i=1}^{2}\left\|\varphi_{i}-\varphi_{h i}\right\|_{h} \rightarrow 0 \quad(\text { by }(d) \text { and }(g)) .\right.
\end{aligned}
$$


On the other hand, [H4] assures that

$$
\begin{aligned}
\| \nabla_{h} u_{h} & +R_{h}\left(\hat{u}_{h}-S_{h} u_{h}\right)\left\|_{\Omega} \leq\right\| \nabla_{h} u_{h}\left\|_{\Omega}+\right\| R_{h}\left(\hat{u}_{h}-S_{h} u_{h}\right) \|_{\Omega} \\
& \leq\left\|\nabla_{h} u_{h}\right\|_{\Omega}+C_{r}\left|\left\{u_{h}, \hat{u}_{h}\right\}\right|_{h} \leq\left(1+C_{r}\right)\left|\left\{u_{h}, \hat{u}_{h}\right\}\right|_{h} \leq\left(1+C_{r}\right)
\end{aligned}
$$

so that the subfamily $\left\{\nabla_{h} u_{h}+R_{h}\left(\hat{u}_{h}-S_{h} u_{h}\right)\right\}_{h>0}$ is uniformly bounded. Thus, there exists its subfamily (denoted by the same notation) that converges weakly in $L^{2}(\Omega)^{2}$ to an element $w \in L^{2}(\Omega)^{2}$.

By these observations and $(g)$, the right-hand side of $(h)$ converges to $(w, \varphi)_{\Omega}$ as $h \downarrow 0$, while the left-hand side converges to $-\left(u_{0}, \operatorname{div} \varphi\right)_{\Omega}$. Thus,

$$
-\left(u_{0}, \operatorname{div} \varphi\right)_{\Omega}=(w, \varphi)_{\Omega}
$$

that is, $w=\nabla u_{0} \in L^{2}(\Omega)^{2}$ in the sense of distribution, and hence $u_{0} \in H^{1}(\Omega)$. Although we selected a subfamily of $\left\{\nabla_{h} u_{h}+R_{h}\left(\hat{u}_{h}-S_{h} u_{h}\right)\right\}_{h>0}$, there was no need of such selection because of the uniqueness of $\nabla u_{0}$ for $u_{0}$.

We have now shown that the whole subfamily $\left\{\nabla_{h} u_{h}+R_{h}\left(\hat{u}_{h}-S_{h} u_{h}\right)\right\}_{h>0}$ converges weakly to $\nabla u_{0}$ in $L^{2}(\Omega)^{2}$, and the final process is to prove $u_{0} \in H_{D}^{1}(\Omega)$. In this respect, we should notice that $(f)$ holds for any $\varphi \in C^{\infty}(\bar{\Omega})^{2}$ with $\left.\varphi\right|_{\partial \Omega \backslash \partial \Omega_{D}}=0$, since $\hat{u}_{h}=0$ on $\partial \Omega_{D}$ and $\varphi=0$ on $\partial \Omega \backslash \partial \Omega_{D}$. Moreover, for such $\varphi$, we can choose an appropriate family of functions $\left\{\varphi_{h}=\left\{\varphi_{h 1}, \varphi_{h 2}\right\} \in\left(Q^{h}\right)^{2}\right\}_{h>0}$ that satisfy $(g)$. Then we have (h) again, and, by taking its limit as $h \downarrow 0$, we find that

$$
-\left(u_{0}, \operatorname{div} \varphi\right)_{\Omega}=\left(\nabla u_{0}, \varphi\right)_{\Omega}, \text { or, by the Green formula, } \int_{\partial \Omega_{D}} u_{0}(\varphi \cdot n) d s=0 .
$$

Noting the arbitrariness of $\varphi$ on $\partial \Omega_{D}$, we can conclude that $u_{0}=0$ on $\partial \Omega_{D}$.

\section{Observations on hypotheses}

We will give some sufficient conditions to assure hypotheses [H0] through [H4] to hold. At present, the conditions to be given are not necessarily satisfactory from both theoretical and practical viewpoints, and there remains much room for improvement.

As is fully discussed in [4], the essential points are approximation capabilities of polynomial functions at element level and some trace theorems for each element under some geometrical conditions on element shapes and sizes. We will explain them below for the following choice of discrete spaces $\left(0<\sigma \leq \frac{1}{2}\right)$ : for given $k \in \mathbf{N}$, let $U^{h}, \hat{U}^{h}$ and $Q^{K}\left(\forall K \in \mathscr{T}^{h}\right)$ be

$$
\begin{aligned}
& U^{h}=\Pi_{K \in \mathscr{T}_{h}} P_{k}(K) \subset H^{\frac{3}{2}+\sigma}\left(\mathscr{T}^{h}\right), Q^{K}=P_{k}(K) \subset L^{2}(K) \text { or } P_{k-1}(K) \subset L^{2}(K), \\
& \hat{U}^{h}=\Pi_{e \in \mathscr{E}_{h}} P_{k}(e) \subset L^{2}\left(\Gamma^{h}\right) \text { or } C\left(\Gamma^{h}\right) \cap \Pi_{e \in \mathscr{E}^{h}} P_{k}(e) .
\end{aligned}
$$

\subsection{Chunkiness condition for star-shaped elements}

To show [H0], we require some regularity conditions on the triangulations to avoid too thin elements. There have been proposed a number of statements relevant to this issue, among which we here employ the chunkiness condition of Deny-Lions and Brenner-Scott [1,9] for convenience. To such an end, we assume that each $K \in \mathscr{T}^{h}$ is star-shaped with respect to a closed disk $D_{K} \subset K$ of positive radius, and then define $\rho_{K}$ as the supermom of radii of such possible $D_{K}$ 's. Then, by using $\rho_{K}$ and $h_{K}=\operatorname{diam} K$, the chunkiness parameter $\zeta_{K}$ for $K \in \mathscr{T}^{h}$ is defined as :

$$
\zeta_{K}=h_{K} / \rho_{K}
$$

The chunkiness condition for the family $\left\{\mathscr{T}^{h}\right\}_{h>0}$ is now stated as follows. 
Chunkiness condition For $\left\{\mathscr{T}^{h}\right\}_{h>0}$, there exists a positive constant $\gamma_{C}$ such that

$$
\sup _{\mathscr{T} h} \max _{K \in \mathscr{T} h} \zeta_{K} \leq \gamma_{C}
$$

Remark 3. If $K$ is a triangle, this condition coincides with the minimum angle one [9, 11]. By (32), too thin elements are excluded from $\left\{\mathscr{T}^{h}\right\}_{h>0}$. On the other hand, non-convex finite elements are allowed to a certain extent, although convexity of elements is sometimes essential in classical FEM [11]. From (33), we have for example $C_{1} h_{K}^{2} \leq|K| \leq C_{2} h_{k}^{2}$ for all $h>0$ and $K \in \mathscr{T}^{h}$ with appropriate $C_{1}$ and $C_{2}$.

Under the above condition, we can show the following approximation capabilities of $U^{h}:$ for $v \in H^{\frac{3}{2}+\sigma}\left(\mathscr{T}^{h}\right)(0<$ $\sigma<\frac{1}{2}$ ), it holds that

$$
\inf _{v_{h} \in U^{h}}\left[h^{-1}\left\|v-v_{h}\right\|_{\Omega}+\left\|\nabla_{h}\left(v-v_{h}\right)\right\|_{\Omega}\right] \leq C^{\frac{1}{2}+\sigma}\|v\|_{H^{\frac{3}{2}+\sigma}\left(\mathscr{T}^{h}\right)} .
$$

See e.g. $[1,9]$ for the details, and similar results in the mesh-dependent norm $\|\cdot\|_{h}$ are available for $Q^{h}$. Such estimates essentially contribute to assuring [H0].

\subsection{Triangle condition}

To obtain desirable trace theorems for each element, we here assume a kind of cone condition [5, 15].

Triangle condition Let $T_{0}$ be an isosceles triangle with unit base length and height $\gamma_{T}$. Then, for all $h>0, K \in \mathscr{T}^{h}$ and $e \in \mathscr{E}^{K}$, there exists an isosceles triangle $T_{K, e}$ contained in $K$, whose base coincides with $e$ and whose height is $\gamma_{T}|e|\left(T_{K, e}\right.$ is similar to $T_{0}$ with similarity ratio $|e|$, see Fig. 3).

For $T_{0}$ above, we have the trace theorem of form : any $v \in H^{\frac{1}{2}+\sigma}\left(T_{0}\right)\left(0<\sigma \leq \frac{1}{2}\right)$ satisfies

$$
|v|_{\partial T_{0}} \leq C\left(\|v\|_{T_{0}}+|v|_{\frac{1}{2}+\sigma, T_{0}}\right)
$$

where $C>0$ depends only on $\gamma_{T}$ and $\sigma$ [4]. Then introducing an appropriate similarity transformation between $T_{0}$ and $T_{K, e}$ of $K \in \mathscr{T}^{h}$, we easily obtain

$$
|v|_{e} \leq|v|_{\partial K} \leq C\left(|e|^{-\frac{1}{2}}\|v\|_{K}+|e|^{\frac{1+2 \sigma}{4}}|v|_{\frac{1}{2}+\sigma, K}\right) .
$$
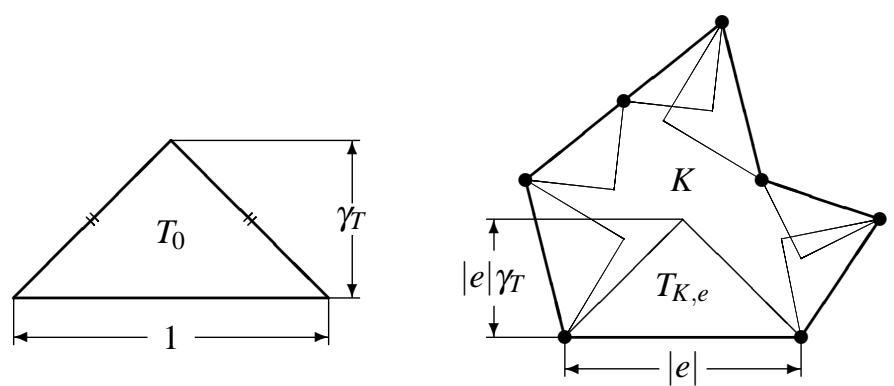

Figure 3: Triangle condition

\subsection{Local quasi-uniformity condition on edge sizes}

Although we do not employ the quasi-uniformity [9] of the family $\left\{\mathscr{T}^{h}\right\}_{h>0}$ here, we still assume that the sizes of edges for each $K \in \mathscr{T}^{h}$ are comparable. 
Local quasi-uniformity of edge sizes $h>0$ and $K \in \mathscr{T}^{h}$,

For $\left\{\mathscr{T}^{h}\right\}_{h>0}$, there exists a positive constant $\gamma_{U}$ such that, for all

$$
\frac{\max _{e \in \mathscr{E} K}|e|}{\min _{e \in \mathscr{E} K}|e|} \leq \gamma_{U}
$$

Remark 4. Since $3 \leq m \leq M$ and $|\partial K|=\sum_{e \in \mathscr{E}_{K}}|e| \geq 2 h_{K}$, the present condition assures that $\min _{e \in \mathscr{E}^{K}}|e| \geq$ $C h_{K}$ for a constant $C>0$ dependent only on $M$ and $\gamma_{U}$.

By this condition, we can modify (36) as, with $C$ slightly changed,

$$
|v|_{e} \leq|v|_{\partial K} \leq C\left(\left|h_{K}\right|^{-\frac{1}{2}}\|v\|_{K}+\left|h_{K}\right|^{\frac{1+2 \sigma}{4}}|v|_{\frac{1}{2}+\sigma, K}\right) .
$$

By (34) and the above, we can deduce [H0] through [H4] completely as in [4], so that we omit the proofs.

\section{Fundamental applications}

The obtained results can be applied to numerical analysis of various problems, but here we list up some very fundamental problems only:

1. Numerical analysis of resolvents and non-coercive problems.

2. Numerical analysis of spectral problems.

3. Derivation of the discrete Poincaré-Friedrichs inequalities.

4. Approximation of problems with non-smooth solutions.

5. Numerical analysis of some nonlinear problems.

As is well known, the first three are closely related to each other [3, 8, 10]. For example, some discrete compactness properties can be derived from the discrete Poincaré-Friedrichs inequalities, see e.g. [3, 6].

As a simple example of problem for item 4, let us consider the problem : given $\tilde{f}=\left\{f_{1}, f_{2}\right\} \in L^{2}(\Omega)^{2}$, find $u \in H_{D}^{1}(\Omega)$ s.t.

$$
(\nabla u, \nabla v)_{\Omega}+(u, v)_{\Omega}=(\tilde{f}, \nabla v)_{\Omega} ; \forall v \in H_{D}^{1}(\Omega),
$$

where the term $(u, v)_{\Omega}$ can be omitted if $\partial \Omega_{D} \neq \emptyset$. Clearly, the solution of this problem may not have additional smoothness such as $u \in H^{1+\varepsilon}(\Omega)$ for some $\varepsilon>0$. A possible example of discretized problem for (39) is : given $\tilde{f}=\left\{f_{1}, f_{2}\right\} \in L^{2}(\Omega)^{2}$, find $\left\{u_{h}, \hat{u}_{h}\right\} \in V_{D}^{h}(\Omega)$ s.t.

$$
\begin{aligned}
B_{h}\left(\left\{u_{h}, \hat{u}_{h}\right\},\left\{v_{h}, \hat{v}_{h}\right\}\right)+\left(u_{h}, v_{h}\right)_{\Omega}=\left(\tilde{f}, \nabla_{h} v_{h}+R_{h}\left(\hat{v}_{h}-S_{h} v_{h}\right)\right)_{\Omega} ; \\
\forall\left\{v_{h}, \hat{v}_{h}\right\} \in V_{D}^{h} .
\end{aligned}
$$

By using Theorem 1, we can show, for example, strong convergence of $\nabla_{h} u_{h}+R_{h}\left(\hat{u}_{h}-S_{h} u_{h}\right)$ to $\nabla u$ in $L^{2}(\Omega)^{2}$ as well as that of $u_{h}$ to $u$ in $L^{2}(\Omega)$ as $h \downarrow 0$, provided that the set of sufficiently smooth function in $H_{D}^{1}(\Omega)$ is dense there. We omit the proof, but it can be performed by essentially the same ideas and techniques as in the proof of Theorem 1 .

As an example for the final item, we can consider the boundary value problems for a simple semi-linear elliptic equation $-\Delta u=f(u)$, where $f(\cdot)$ is a polynomial function. For the analysis of the corresponding approximate problems discretized by DGFEM, we need additional results on some function spaces such as $L^{p}(\Omega)(p \neq 2)$, so that the analysis in e.g. [6] may be effective.

\section{Concluding remarks}

We have discussed a Rellich-type discrete compactness for some DGFEM. Our approach has limitations in the framework of Hilbert methods, but may be effective for some purposes. We can generalize the present methodologies to wider classes of DGFEM, but we have not attempted such generalization to make the 
description concise. Extensions to 3D cases appears to be possible, provided that the regularity results of the corresponding auxiliary problem are well established and the appropriate conditions for triangulations are found. As was already mentioned, we can apply the results to various problems, and such applications will be reported elsewhere.

Acknowledgements The author would like to thank Prof. B. Cockburn, University of Minnesota, for fruitful discussions on hybridized DGFEM during his stay at University of Tokyo. The comments by Prof. D. N. Arnold, University of Minnesota, at Mini-Workshop on Numerical Analysis held at Hakone, Japan, was also very valuable.

\section{References}

[1] Apel, T.: Interpolation in $h$-version finite element spaces. In: Stein, E., de Borst, R., Hughes, T.J.R. (eds.) Encyclopedia of Computational Mechanics, Vol. 1, John Wiley \& Sons, Chichester (2004)

[2] Arnold, D.N.: An interior penalty finite element method with discontinuous elements. SIAM J. Numer. Anal. 19, 742-760 (1982)

[3] Antonietti, P.F., Buffa, A., Perugia, I.: Discontinuous Galerkin approximation of the Laplace eigenproblem. Comput. Methods Appl. Mech. Engrg. 195, 3483-3503 (2006)

[4] Arnold, D.N., Brezzi,F., Cockburn, B., Marini, L.D.: Unified analysis of discontinuous Galerkin methods for elliptic problems. SIAM J. Numer. Anal. 39, 1749-1779 (2002)

[5] Adams, R.A., Fournier, J.J.F.: Sobolev Spaces. 2nd ed. Academic Press, Amsterdam (2003)

[6] Buffa, A., Ortner, C.: Compact embeddings of broken Sobolev spaces and applications. IMA J. Numer. Anal. 29, 827-855 (2009)

[7] Boffi, D.: Fortin operator and discrete compactness for edge elements. Numer. Math. 87, 229-246 (2000)

[8] Boffi, D.: Approximation of eigenvalues in mixed form, discrete compactness property, and application to $h p$ mixed finite elements. Computer Methods in Applied Mechanics and Engineering. 196, 3672-3681 (2007)

[9] Brenner, S.C., Scott, L.R.: The Mathematical Theory of Finite Element Methods, 3rd ed. SpringerVerlag, New York (2008)

[10] Chatlin, F.: Spectral Approximation of Linear Operators. Academic Press, New York (1983)

[11] Ciarlet, P.G.: The Finite Element Method for Elliptic Problems, 2nd ed. SIAM, Philadelphia (2002)

[12] Cockburn, B., Gopalakrishnan, J., Lazarov, R.: Unified hybridization of discontinuous Galerkin, mixed, and continuous Galerkin methods for second order elliptic problems. SIAM J. Numer. Anal. 47, 13191365 (2009)

[13] Cockburn, B., Guzmán, J., Soon, S.-C., Stolarski, H.K.: An analysis of the embedded discontinuous Galerkin method for second-order elliptic problems. SIAM J. Numer. Anal. 47, 2686-2707 (2009)

[14] Fung, Y.C., Tong, P.: Classical and Computational Solid Mechanics. World Scientific Pub., Singapore (2001)

[15] Grisvard, P.: Elliptic problems in nonsmooth domains. Pitman, Boston (1985)

[16] Hesthaven, J.S., Warburton, T.: Nodal Discontinuous Galerkin Methods: Algorithms, Analysis, and Applications. Springer-Verlag, New York (2008)

[17] Jamet, P.: Estimation of the interpolation error for quadrilateral finite elements which can degenerate into triangles. SIAM J. Numer. Anal. 14, 925-930 (1977) 
[18] Kikuchi, F.: On a discrete compactness property for the Nedelec finite elements. J. Fac. Sci., The University of Tokyo, Sec. IA, Mathematics. 36, 479-490 (1989)

[19] Kikuchi, F.: Theoretical analysis of Nedelec's edge elements. Japan J. Industr. Appl. Math. 18, 321-333 (2001)

[20] Kikuchi, F., Ando, Y.: A new variational functional for the finite-element method and its application to plate and shell problems. Nucl. Eng. Des. 21, 95-113 (1972)

[21] Kikuchi, F., Ando, Y.: Some finite element solutions for plate bending problems by simplified hybrid displacement method. Nucl. Eng. Des. 23, 155-178 (1972)

[22] Kikuchi, F., Ishii, K., Oikawa, I.: Discontinuous Galerkin FEM of hybrid displacement typedevelopment of polygonal elements —. Theo. \& Appl. Mech. Japan. 57, 395-404 (2009)

[23] Komzsik, L.: What Every Engineer Should Know about Computational Techniques of Finite Element Analysis, 2nd ed. CRC Press, New York (2009)

[24] Li, B.Q.: Discontinuous Finite Elements in Fluid Dynamics and Heat Transfer. Springer-Verlag, London (2006)

[25] Mang, H.A., Gallagher, R.H.: A critical assessment of the simplified hybrid displacement method. Int. J. Num. Meth. Eng. 11, 145-167 (1977)

[26] Oikawa, I.: Hybridized discontinuous Galerkin method with lifting operator. JSIAM Letters. 2, 99-102 (2010)

[27] Oikawa, I. Kikuchi, F.: Discontinuous Galerkin FEM of hybrid type. JSIAM Letters. 2, 49-52 (2010)

[28] Pian, T.H.H., Wu, C.-C.: Hybrid and Incompatible Finite Element Methods", Chapman \& Hall, Boca Raton (2005)

[29] Quek, S.S., Liu, G.R.: Finite Element Method: A Practical Course. Butterworth-Heinemann, Oxford (2003)

[30] Stummel, F.: Basic compactness properties of nonconforming and hybrid finite element spaces. RAIRO, Anal. Numér. 14, 81-115 (1980)

[31] Tong, P.: New displacement hybrid finite element models for solid continua. Int. J. Num. Meth. Eng. 2, 73-83 (1970)

[32] Zhang, J., Kikuchi, F.: Interpolation error estimates of a modified 8-node serendipity finite element. Numer. Math. 85, 503-524 (2000) 